\title{
PluS Nanoparticles Loaded with Sorafenib: Synthetic Approach and Their Effects on Endothelial Cells
}

\author{
Francesco Palomba, ${ }^{\dagger}$ Damiano Genovese, ${ }^{\dagger} \odot$ Enrico Rampazzo, ${ }^{\dagger}$ Nelsi Zaccheroni, ${ }^{\dagger}$ Luca Prodi,,${ }^{\dagger}{ }^{\dagger} \odot$ \\ and Lucia Morbidelli*,
}

†Dipartimento di Chimica “Giacomo Ciamician”, Alma Mater Studiorum, Università di Bologna, Via Selmi 2, 40126 Bologna, Italy

${ }^{\ddagger}$ Dipartimento di Scienze della Vita, Università di Siena, Via A. Moro 2, 53100 Siena, Italy

\section{Supporting Information}

\begin{abstract}
Silica nanostructures are widely investigated for theranostic applications since relatively mild and easy synthetic methods allow the fabrication of multicompartment nanoparticles (NPs) and fine modulation of their properties. Here, we report the optimization of a synthetic strategy leading to brightly fluorescent silica NPs with a high loading ability, up to 45 molecules per NP, of Sorafenib, a small molecule acting as an antiangiogenic drug. We demonstrate that these NPs can efficiently release the drug and they are able to inhibit endothelial cell proliferation and migration and network formation. Their lyophilization can endow them with long shelf stability, whereas, once in solution, they show a much slower release compared to analogous micellar systems. Interestingly, Sorafenib released from Pluronic silica NPs completely prevented endothelial cell responses and postreceptor mitogen-activated protein kinase signaling ignited by vascular

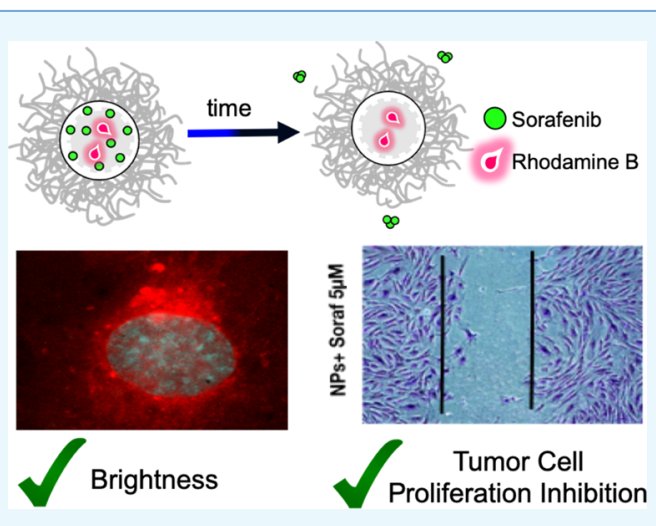
endothelial growth factor, one of the major players of tumor angiogenesis. Our results indicate that these theranostic systems represent a promising structure for anticancer applications since NPs alone have no cytotoxic effect on cultured endothelial cells, a cell type to which drugs and exogenous material are always in contact once delivered.
\end{abstract}

\section{INTRODUCTION}

Vascular endothelial cells (ECs) are the first-line barrier for injected drugs or medical devices as nanoparticles (NPs). It is thus mandatory to ascertain the safety and lack of toxicity of synthetic materials even before the study of their therapeutic efficacy.

In the research discussed in this paper, we have concentrated our attention on Sorafenib, a small molecule acting as an antiangiogenic drug, able to inhibit the kinase moiety of the vascular endothelial growth factor receptor (VEGFR)-2 and other kinases. 'Vascular endothelial growth factor (VEGF) plays an important role in tumor development, invasion, and metastasis by promoting tumor angiogenesis. ${ }^{2,3}$ Several malignancies are treated through the use of VEGFR tyrosine kinase inhibitors (TKIs). ${ }^{4}$ In particular, Sorafenib is an orally active multi-TKI that targets VEGFR-2 and -3, platelet-derived growth factor receptor $\beta$, and Raf kinase, all of which have been implicated in neovascularization and tumor progression. ${ }^{5,6}$ The Food and Drug Administration has approved the use of Sorafenib for patients with renal cell carcinoma and hepatocellular carcinoma, and the list of indications for this drug is growing. ${ }^{7-12}$ Although remarkably well tolerated by patients, Sorafenib exhibits a distinct pattern of adverse events (AEs) that are thought to be related to the inhibition of angiogenesis. Potentially, life-threatening AEs associated with Sorafenib treatment include hypertension, hemorrhage, and arterial and venous thromboembolism. ${ }^{13-17}$ Hemorrhagic events have major negative impacts on mortality, morbidity, and healthcare costs, often leading to treatment delays and interruptions. $^{17,18}$

An interesting general approach to limit anticancer drug AEs is to design new formulations for their specific targeting and slow drug release. In the last decades, several reports demonstrated that nanotechnology can provide a promising platform in medicine for carrying and delivering chemotherapeutic drugs by reducing side effects, increasing drug accumulation at the tumor site, and improving blood circulation.

Nanoparticles have been extensively applied in cancer diagnosis and treatment exploiting their potentialities in single or multidrug delivery. ${ }^{19}$ Therapeutic NPs can be designed to accumulate at the tumor tissue/organ, where the active agent is released, increasing the therapeutic efficacy by reducing the dose, the incidence of the treatment, and the pernicious effect on healthy sites. Moreover, once administered, NPs have the intrinsic and general tendency to accumulate in the tumor tissue/organ compared with healthy tissues. ${ }^{20}$ This tendency is based on the enhanced permeability and retention (EPR) effect. EPR is a consequence of the fact that solid tumors are characterized by leaky blood vessels and hypoxia, which lead to

Received: June 10, 2019

Accepted: July 18, 2019

Published: August 14, 2019 
the extravasation of NPs. ${ }^{21}$ This effect prolongs the retention of nanomaterials within the pathological organ.

In addition to the EPR effect, nanomaterials can be also engineered with functional groups on their surface (for example, folic acid and hyaluronic acid) to obtain a more specific target toward the tumor organs. ${ }^{22}$

One of the most interesting and promising chemical matrixes used to develop theranostic nanostructures is silica since its synthetic methods allow the fabrication of complex nanoarchitectures where size and surface properties can be modulated to control possible interactions with biomolecules, a promising efficient cell internalization, and specific tissue accumulation. ${ }^{23}$ Their versatile synthesis can allow obtaining structures with different sizes ( $5 \mathrm{~nm}$ to $1 \mu \mathrm{m})$, porosities, and shapes (such as disk, rod, spherical particles, hollow sphere). ${ }^{24,25}$ Several different morphologies of silica nanoparticles are under investigation in clinical and preclinical tests, aimed at the evaluation of the cytotoxicity and the biocompatibility by oral or subcutaneous administration. ${ }^{26}$ Furthermore, the possibility to obtain nanostructures that can be broken by suitable stimuli $(\mathrm{pH}$, reducing agents, light) inside cells and tissues can allow silica NPs to properly modify drug delivery and excretion processes, increasing their efficacy and reducing even more their long-term risks. ${ }^{27,28}$

In this context, we recently developed the synthesis of multicompartment silica core/poly(ethylene glycol) (PEG) shell nanoparticles, named Pluronic silica (PluS) NPs, obtained with a one pot direct micelles template approach, developing many bright luminescent materials ${ }^{29,30}$ for applications in in vitro $^{31}$ and in vivo ${ }^{32,33}$ optical and photoacoustic ${ }^{34}$ imaging as chemosensors $^{35}$ or as electrochemiluminescent probes. ${ }^{36}$ Interestingly, all of the experiments performed so far in vitro on several cell lines and in vivo showed the absence of toxicity in the useful concentration range. ${ }^{31}$

To optimize the advantages and efficacy for the application of these PluS NPs in vivo, we are interested to develop suitable strategies for the obtainment of theranostic materials, with photothermal therapy, ${ }^{34}$ drug delivery, and optical imaging capabilities. In this context, we present here the design of a modified synthetic approach of these luminescent NPs conceived to entrap a large number of Sorafenib molecules while keeping release kinetics that could be compatible with an in vivo use.

In addition, we present also a modification of the overall formulation adopted to maintain the property of nanoparticles, including drug content and colloidal stability, stable over time with a long shelf time. To obtain this goal, freeze-drying, also known as lyophilization, is one of the most used techniques. Freeze-drying is a process that consists of removing water from a frozen sample by sublimation and desorption under vacuum. Nevertheless, this process generates various stresses during freezing and drying steps; for this reason, species protectants, such as monosaccharides, oligosaccharides, proteins, or other polymers [PEG, poly(vinylpyrrolidone) (PVP)], are usually added to the formulation to protect the nanoparticles from freezing and desiccation stresses. ${ }^{37}$ In particular, freeze-drying has been considered as a good technique to improve the longterm stability of colloidal nanoparticles, especially aimed at drug delivery purposes since dehydration can avoid drug leaks from the nanocarriers. The main goal for a freeze-drying process applied to theranostic nanoparticles is to obtain welldispersible nanocarriers, stable over time and moreover after rehydration. This is a key factor for the application of freeze- dried nanoarchitectures in a biological environment since colloidal properties of the carrier affect the behaviors during the application. ${ }^{38,39}$ The strategy that we propose here is based on the use of PVP as a protectant to yield one of the smallest $\mathrm{PEG} /$ silica nanoarchitectures as a potential nanocarrier for Sorafenib with a simple preparation, high fluorescence brightness, and long-term colloidal stability. ${ }^{40}$

The aim of the study was twofold: (i) to evaluate PluS NPs safety on endothelial cells in basal conditions (1\% serum) and after stimulation ( $5 \%$ serum) and (ii) to characterize the effect of Sorafenib released by these NPs on endothelial cell morphology, viability, migration, and VEGF-induced angiogenesis-related functions.

\section{RESULTS AND DISCUSSION}

Design of the Synthesis of Dye-Doped PluS NPs Loaded with Sorafenib. A possible strategy for the entrapment of lipophilic compounds by PluS NPs ${ }^{46,47}$ is based on the presence, in these multicompartment structures, of a lipophilic task close to the surface of the silica core. ${ }^{48}$ In this way, we were able to obtain efficient chemosensors, electrochemiluminescence labels, ${ }^{36}$ and photoswitching nanostructures. ${ }^{49}$ As a first step, we tried to follow this strategy for the inclusion of Sorafenib, but this approach was unsatisfactory since the loading resulted to be relatively low $(<10$ drug molecules per NP). Another embedding strategy is based on the insertion of the active species in the nanoparticle core during the synthesis. The first synthetic step for the obtainment of PluS NPs is the formation of Pluronic F-127 micelles; the inclusion of Sorafenib into the micelle dispersion led, in few minutes, to the complete dissolution of the drug, otherwise insoluble in water. In the standard synthetic procedure, this is followed by a second step in which the silica precursor used for the formation of the PluS NPs, tetraethylorthosilicate (TEOS), ${ }^{31}$ undergoes condensation in the acidic aqueous media. ${ }^{43}$ This synthetic strategy led again to a quite low Sorafenib entrapment in the NPs, which we attributed to a not sufficiently fast (hours) hydrolysis kinetic of the ethoxysilane derivative in these conditions. This finding prompted us to substitute TEOS with tetramethoxyorthosilicate (TMOS), which is more reactive toward hydrolysis and condensation and can react completely in a shorter timeframe.

To obtain fluorescent NPs, we inserted at this stage a trimethoxysilane derivative of rhodamine $\mathrm{B}(\mathrm{RB})$ to covalently attach the dye to the silica matrix (Figure 1). Also, in this case, the choice of the derivative was adopted because of its faster kinetics; the use of the analogous triethoxysilane derivative lead to a 5-fold smaller doping degree (data not shown). It is important to underline that the use of rhodamine was made here to optimize the performances of the NPs for in vitro

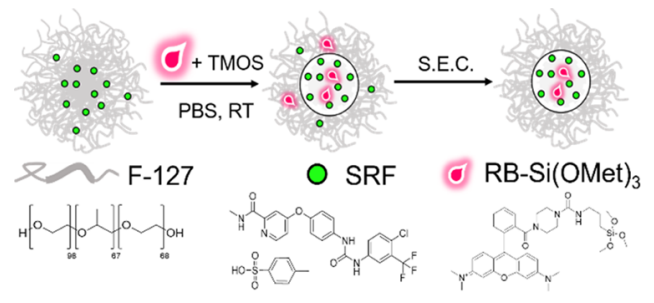

Figure 1. Schematic representation of the synthesis of Sorafenib tosylate (SRF)-loaded Pluronic F-127 silica nanoparticles functionalized with rhodamine. 
Table 1. Amount of Reagents and Main Morphological and Photophysical Properties of Lyophilized Nanoparticles

\begin{tabular}{|c|c|c|c|c|c|c|c|c|c|c|c|c|}
\hline name & F-127 (mg) & $\begin{array}{l}\text { SRF } \\
(\mathrm{mg})\end{array}$ & $\begin{array}{c}\text { TMOS } \\
(\mu \mathrm{L})\end{array}$ & $\begin{array}{l}\text { PBS } \\
(\mu \mathrm{L})\end{array}$ & RB-TMS (mg) & $\begin{array}{c}\text { no of } \\
\mathrm{RB} / \mathrm{NP}^{a}\end{array}$ & $\begin{array}{c}\text { no of } \\
\text { SRF/NP }\end{array}$ & $d_{\mathrm{H}}(\mathrm{nm})+\mathrm{SD}$ & PDI & $\begin{array}{c}\lambda_{\text {max,abs }} \\
(\mathrm{nm})\end{array}$ & $\begin{array}{c}\lambda_{\text {max,em }} \\
(\mathrm{nm})\end{array}$ & $\Phi_{\mathrm{F}}^{b}$ \\
\hline NPs & 200 & & 250 & 3200 & 0.8 & 6 & & $30 \pm 5$ & 0.352 & 562 & 591 & 0.20 \\
\hline SRF-NPs & 200 & 19 & 250 & 3200 & 0.8 & 6 & 45 & $32 \pm 6$ & 0.430 & 563 & 590 & 0.21 \\
\hline
\end{tabular}

${ }^{a}$ Measured by absorption spectra. ${ }^{b}$ Measured using rhodamine B in ethanol $\left(\Phi_{\text {ref }}=0.96\right)$ as the reference.

studies since microscopes are seldom equipped for nearinfrared (NIR) light excitation and detection. However, the versatility of the synthesis of PluS NPs opens up the possibility to use a wide range of dyes including NIR ones, as cyanines, that could allow for in vivo fluorescence imaging, photoacoustic imaging, and photothermal therapy. ${ }^{29-34}$

The importance to limit the release of Sorafenib during synthesis suggested also to change the purification system, moving from dialysis $(\sim 1$ day) to a much faster size exclusion chromatography (SEC) separation (Sephadex G-25), using phosphate-buffered saline (PBS) as the mobile phase. From transmission electron microscopy images [see the Supporting Information (SI)], it is possible to observe that the diameter of the silica core is $10 \pm 1 \mathrm{~nm}$, whereas the hydrodynamic diameter obtained with dynamic light scattering (DLS) measurements (see SI) was $30 \mathrm{~nm}$. This value is slightly larger than the usual hydrodynamic diameter measured for PluS NPs $(25 \mathrm{~nm})$, probably because of the use of TMOS instead of TEOS. The possible limitation related to the storage of PluS NPs stock dispersion, in which Sorafenib can leach from the NPs, has been addressed, optimizing a lyophilization protocol (reported in the Experimental Section) to obtain PluS NPs in the form of a "ready to use" water-dispersible solid powder. The choice of PVP K30 as the protecting agent was motivated by the fact that it is a water soluble, cheap, and nontoxic polymer for the efficient passivation of surfaces. In addition, PVP was preliminarily tested to assess its potential cytotoxic effect. Human umbilical vein endothelial cells (HUVECs) were exposed to PVP concentrations ranging from 1 to $100 \mathrm{nM}$, in excess with respect to the final formulation. Our experiments did not evidence any toxic effect on cell viability (see SI, p S7). This step provided a formulation of SRF-PluS NPs with long-term shelf stability that is not affected by any drug release after preparation. The final averaged dye doping degree consists of six rhodamine $B$ moieties, conferring to the NPs a quite high brightness $\left(\varepsilon_{\mathrm{NP}, 565 \mathrm{~nm}}=6 \times 10^{5} \mathrm{M}^{-1} \mathrm{~cm}^{-1} ; \Phi_{\mathrm{F}}=0.21\right.$, Table 1$)$, making them suitable for optical imaging. As far as the drug loading was concerned, each NP was able to host 45 Sorafenib molecules (Table 1), a very significant amount considering the small total volume of the nanostructure. After this preparation, $1 \mathrm{mg}$ of lyophilized solid corresponds to $25 \mu \mathrm{g}$ of Sorafenib. This formulation was used for all of the following experiments.

Sorafenib Release and Nanoparticle Stability. The absorption spectrum of Sorafenib inside the nanoparticles presents a very similar profile to the one shown by the drug in methanol solution, with an absorption maximum at $265 \mathrm{~nm}$. As already mentioned, the water solubility of Sorafenib is very poor and precipitation occurs at very low concentration $\left(>10^{-5}\right.$ $M)$. This means that the quantity of Sorafenib that is vehiculated in water by the NPs is higher than its solubility and the drug, when released, precipitates, producing a decrease in its absorbance and an increase in scattering. Therefore, it was possible to investigate its release from the NPs measuring the decrease of absorbance at $265 \mathrm{~nm}$ (Figure 2). This method
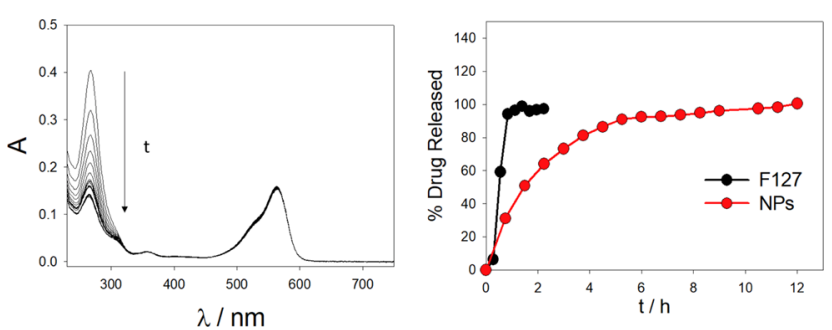

Figure 2. Left: absorbance spectra variation of SRF-PluS NPs $(2.5 \times$ $\left.10^{-7} \mathrm{M}\right)$ during the drug release. Right: trend comparison of the drug release of SRF-F127 micelles vs PluS NPs. PBS pH 7.4, $37^{\circ} \mathrm{C}$.

allows the release kinetics to be directly monitored, and it can be more accurate than other assays, such as dialysis or chromatography, where the separation process can alter the kinetics themselves. ${ }^{10,11}$

The amount of released Sorafenib from PluS NPs to the bulk solution was obtained by the equation

$$
\% \text { release }=\left[1-\left(\frac{A_{265 \mathrm{~nm}}(t)}{A_{265 \mathrm{~nm}}(t=0)}\right)\right] \times 100
$$

The drug molecules entrapped in the PluS NPs diffuse spontaneously and almost quantitatively during $6 \mathrm{~h}$, a much longer time if compared with the few minutes measured for the Pluronic F-127 micelle architecture, at the same Sorafenib concentration $(0.5 \mu \mathrm{M}$, Figure 2$)$.

To verify the stability of the nanoparticles, we monitored their hydrodynamic diameter during the drug release by DLS analysis (Figure 3). The DLS distributions confirm, as expected, that the hydrodynamic diameter remains constant at about $33 \mathrm{~nm}$ over all of the observed period (3 days).

The data presented so far demonstrated that these NPs have suitable stability and drug release capability to allow their potential use as a drug carrier. Therefore, we proceeded with biological tests to investigate the in vitro effects of this nanocarrier preparation on cells present in the tumor microenvironment.

Morphology of HUVEC Following Treatment with NPs. First, we evaluated the cell morphology and cytoskeletal organization of endothelial cells treated or not with PluS NPs. The concentration of NPs was chosen to have a concentration of Sorafenib equal to the one used, as a control, for the free drug. The localization and intensity of the cytoskeletal proteins $\beta$-actin and vimentin were evaluated by immunofluorescence. In the presence of NPs, cell morphology was unaltered with respect to the control conditions ( $1 \%$ serum), demonstrating that NPs alone had no cytotoxic effect (Figure 4A). Immunofluorescence analysis revealed that both in control conditions ( $1 \%$ serum) and in the presence of NPs alone, $\beta$ actin labeling was spread in the cytoplasm and stress fibers could be seen in both conditions (Figure 4B). Accordingly, vimentin intermediate filaments were visible in both conditions (Figure 4C). 


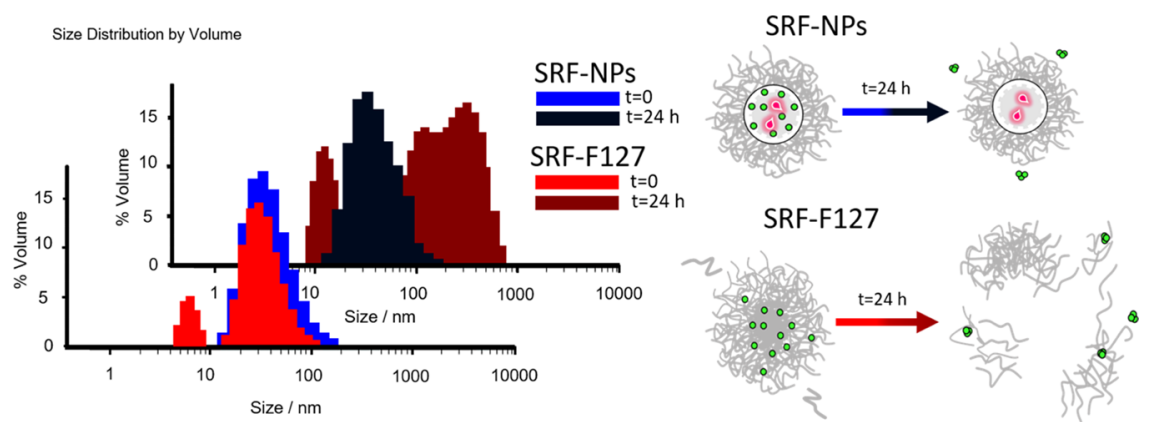

Figure 3. Left: hydrodynamic size distribution of the nanocarriers before and after the drug release (red, SRF-F127; blue, SRF-NPS). Right: schematic representation of the two different mechanisms of the drug reservoir conducted by Pluronic F-127 silica nanoparticles vs Pluronic F-127 micelles.
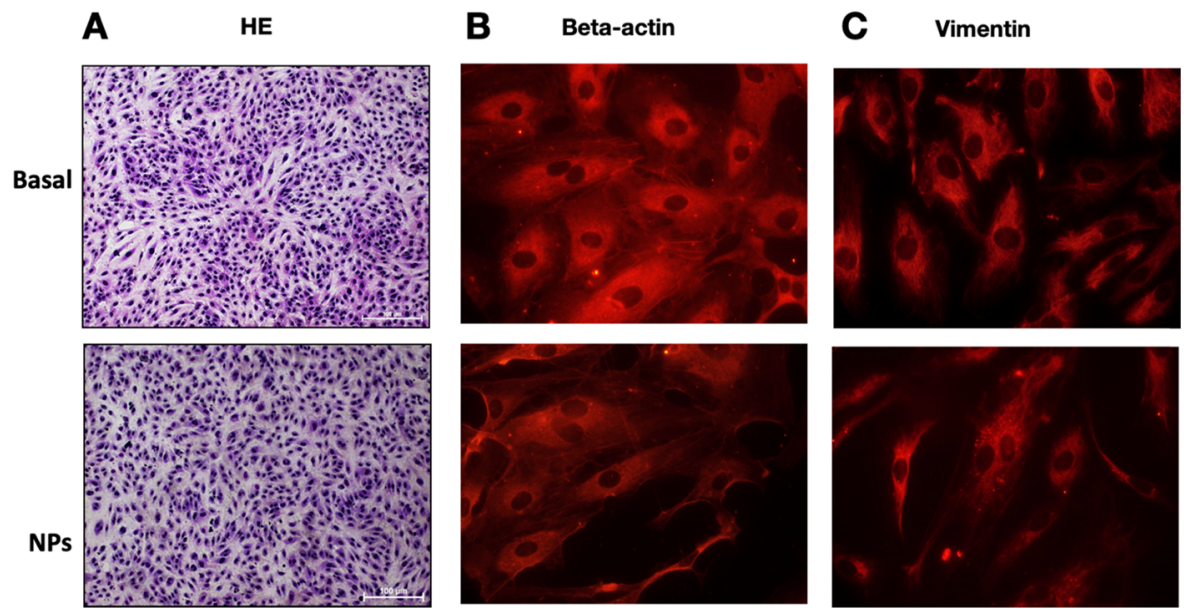

Figure 4. Morphology of HUVEC following treatment with NPs. (A) After $24 \mathrm{~h}$ of cell exposure to NPs (diluted to obtain the similar final concentration of NPs as Sorafenib $5 \mu \mathrm{M}$ loaded NPs, used in the following experiments) in medium containing $1 \%$ serum, cells were fixed, stained, and photographed using the microscope (10X). (B, C) Immunofluorescence for cytoskeletal proteins. Cells, stimulated for $24 \mathrm{~h}$ with NPs (diluted to obtain the similar final concentration of NPs as Sorafenib $5 \mu \mathrm{M}$ loaded NPs) in medium containing $1 \%$ serum, were fixed and immunostained for (B) $\beta$-actin and (C) vimentin. Pictures were taken using the fluorescence microscope $(63 \times)$.

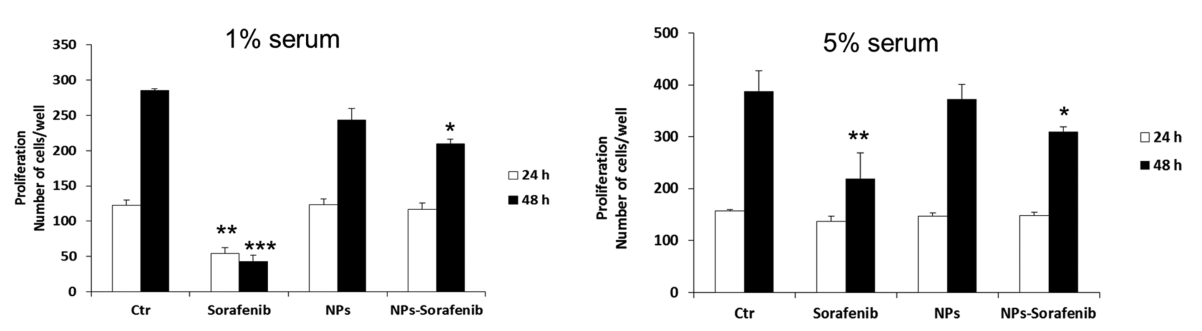

Figure 5. Cell number evaluated in HUVEC treated with Sorafenib $(5 \mu \mathrm{M})$ and NPs and SRF-PluS NPs (diluted to obtain the similar final concentration of Sorafenib) for 24 and $48 \mathrm{~h}$. The experiments were performed in medium containing 1 and $5 \%$ of serum. Data were expressed as cells counted/well \pm standard error $(\mathrm{SE}) . * p<0.05$, $* * p<0.01$, and $* * * p<0.001$ vs control conditions.

Effect of NPs Loaded with Sorafenib on HUVEC Proliferation, Migration, and Cord Formation. Cell survival (evaluated as cell number) in medium with 1 and $5 \%$ fetal bovine serum (FBS) was monitored at 24 and $48 \mathrm{~h}$. In control conditions, 1 and 5\% serum, HUVEC cells increased in number with time. The growth was higher with 5\% FBS. Incubation of the cells with NPs alone or with SRF-PluS NPs had no effect on the cell number, strengthening their nontoxic effect. On the contrary, free Sorafenib (used at the same concentration of the one loaded on NPs) significantly reduced cell growth. The reduction was higher with $1 \%$ serum and after $48 \mathrm{~h}$ incubation (Figure 5).
Inhibition of cell proliferation induced by free Sorafenib was accompanied by an impairment of cell migration evaluated by the scratch assay (Figure 6). In control conditions (1 and 5\% serum added to the medium) and in the presence of NPs, the wounds completely healed. On the contrary, free Sorafenib inhibited cell migration. When Sorafenib was loaded on NPs, the impairment of cell migration was lower (Figure 6).

Finally, cord formation on Matrigel was evaluated as a morphometric assay of in vitro angiogenesis. While in the basal condition of untreated cells HUVEC was organized in cords and networks with interconnected circles, in all of the other conditions (free Sorafenib, NPs alone, and SRF-PluS NPs), the formation of circles was completely blunted (Figure 7). 


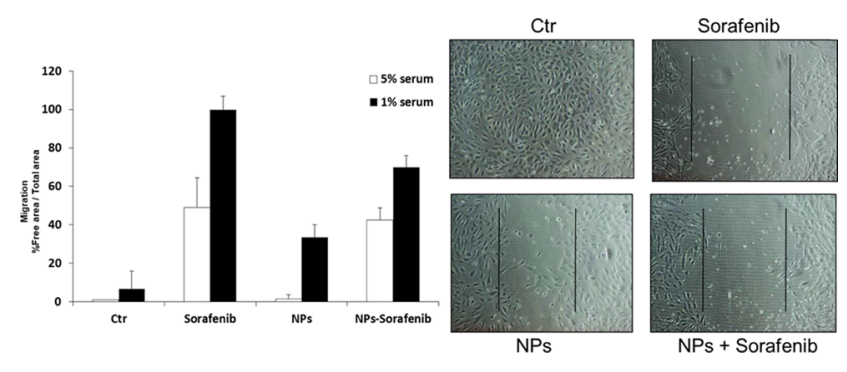

Figure 6. Cell migration was evaluated using the scratch assay on HUVEC treated with Sorafenib $(5 \mu \mathrm{M})$ and NPs and SRF-PluS NPs (diluted to obtain the similar final concentration of Sorafenib). The experiments were performed in medium containing 1 and 5\% of serum. Representative pictures of wounded cell monolayers are shown on the right. The bar graph represents the quantification of cell migration expressed as the percentage of the wound area.
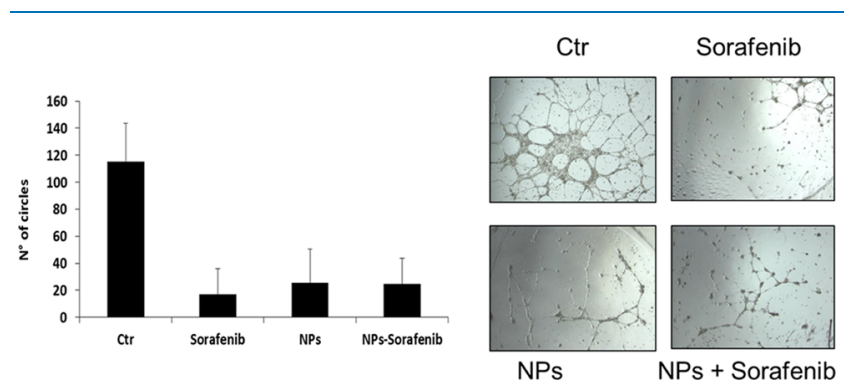

Figure 7. Morphogenic assay on Matrigel was performed on HUVEC treated with Sorafenib $(5 \mu \mathrm{M})$ and NPs and SRF-PluS NPs (diluted to obtain the similar final concentration of Sorafenib) in medium containing 5\% FBS. Representative pictures of network organization are shown on the right. The bar graph represents the quantification of the number of circles monitored after $18 \mathrm{~h}$.

From these series of data obtained on freshly prepared and loaded NPs, nontoxic per se, we can hypothesize that the Sorafenib inhibitory effect on endothelial cells (both quiescent and stimulated with serum) is reduced when the drug is loaded on silica NPs, from which it is released partially and slowly during time, with respect to the free drug, which massively blunts the responses of endothelial cells.

Effect of NPs Loaded with Sorafenib on VEGFInduced Angiogenic Activity in HUVEC. Next, the antiangiogenic effects of Sorafenib-loaded NPs were assayed in different in vitro assays and in the molecular readout of VEGF-induced receptor activation, namely, phosphorylation of ERK1/2. First, the antiproliferative effect of Sorafenib was tested on endothelial cells exposed to VEGF $(25 \mathrm{ng} / \mathrm{mL})$ in medium with $0.1 \%$ serum. In these conditions, after $24 \mathrm{~h}$, VEGF doubled the number of cells. This effect was inhibited by free Sorafenib, NPs, and SRF-PluS NPs, in a similar manner. NPs alone tended to have some inhibitory activity per se (Figure 8A).

The effect of SRF-PluS NPs was tested on HUVEC migration induced by VEGF $(25 \mathrm{ng} / \mathrm{mL})$ in medium containing $0.1 \%$ FBS. Similar to proliferation, the inhibitory effect on VEGF-induced HUVEC migration was obtained with Sorafenib released by silica NPs, with NPs alone being inhibitory but less effective (Figure $8 \mathrm{~B}$ ). The inhibitory effect of NPs alone could be due to the experimental condition used to reveal the proangiogenic effect of VEGF, i.e., a very low serum condition.
A

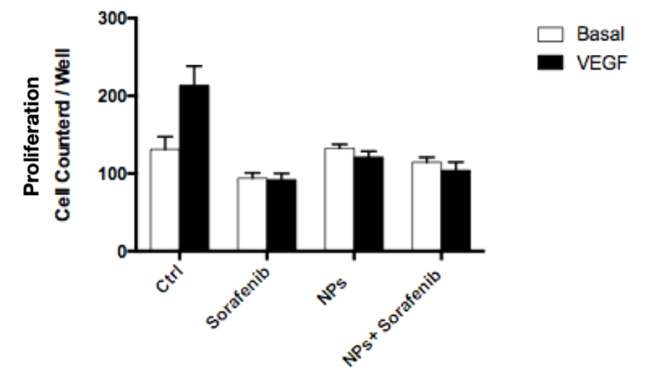

B
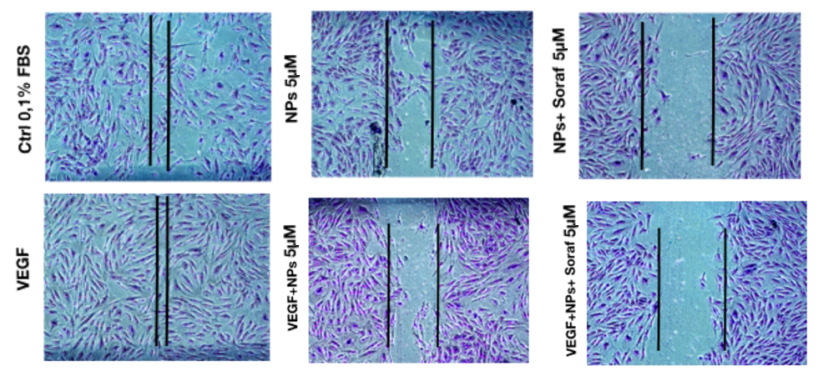

Figure 8. Effect of Sorafenib-loaded NPs on VEGF-induced EC proliferation and migration. (A) Cell number evaluated in HUVEC treated with Sorafenib $(5 \mu \mathrm{M})$ and NPs and SRF-PluS NPs (diluted to obtain the similar final concentration of Sorafenib), with or without VEGF $(25 \mathrm{ng} / \mathrm{mL})$, for $24 \mathrm{~h}$. The experiment was performed in medium containing $0.1 \%$ FBS. Data were expressed as cells counted/ well \pm SE. (B) Cell migration was evaluated using the scratch assay on HUVEC pretreated $(6 \mathrm{~h})$ with NPs and SRF-PluS NPs (diluted to obtain the similar final concentration of Sorafenib $5 \mu \mathrm{M}$ ), with or without VEGF (25 ng/mL); the experiment was performed in medium containing $0.1 \%$ FBS. Representative pictures of wounded cell monolayers are shown.

Next, the potential inhibitory effect of Sorafenib on the molecular readout of VEGF-induced activation in endothelial cells was assessed by means of ERK1/2 phosphorylation (Figure 9). Indeed, VEGFR-2 activation by VEGF in target cells leads to mitogen-activated protein kinase (MAPK) cascade with the final ERK1/2 phosphorylation. Cells were pretreated for $6 \mathrm{~h}$ with the test substances and then stimulated with VEGF $(25 \mathrm{ng} / \mathrm{mL})$ for $10 \mathrm{~min}$. VEGF alone and in the presence of NPs induced a strong ERK1/2 activation, whereas the pretreatment with Sorafenib alone or released by silica NPs completely prevented MAPK signaling ignited by VEGF. The effect of 1 and $5 \mu \mathrm{M}$ Sorafenib released by NPs was similar, documenting that these two concentrations were maximal. Overlapping results were obtained by pretreating the cells with NPs for $1 \mathrm{~h}$, suggesting that $1 \mathrm{~h}$ is enough to release active Sorafenib.

\section{CONCLUSIONS}

In conclusion, we present here a new interesting platform for theranostics that we have designed and prepared, taking advantage of the versatility of the silica synthetic strategies, i.e., fluorescent silica core/PEG shell nanoparticles, named PluS NPs, with a high loading ability of Sorafenib moieties, up to 45 molecules per NP, and with a much slower release compared to the analogous micellar system. An added value is their long shelf stability that has been obtained by an efficient lyophilization of the Sorafenib-loaded NPs (SRF-PluS NPs). Interestingly, we demonstrated that SRF-PluS NPs are able to inhibit cell proliferation, migration, and network formation and 

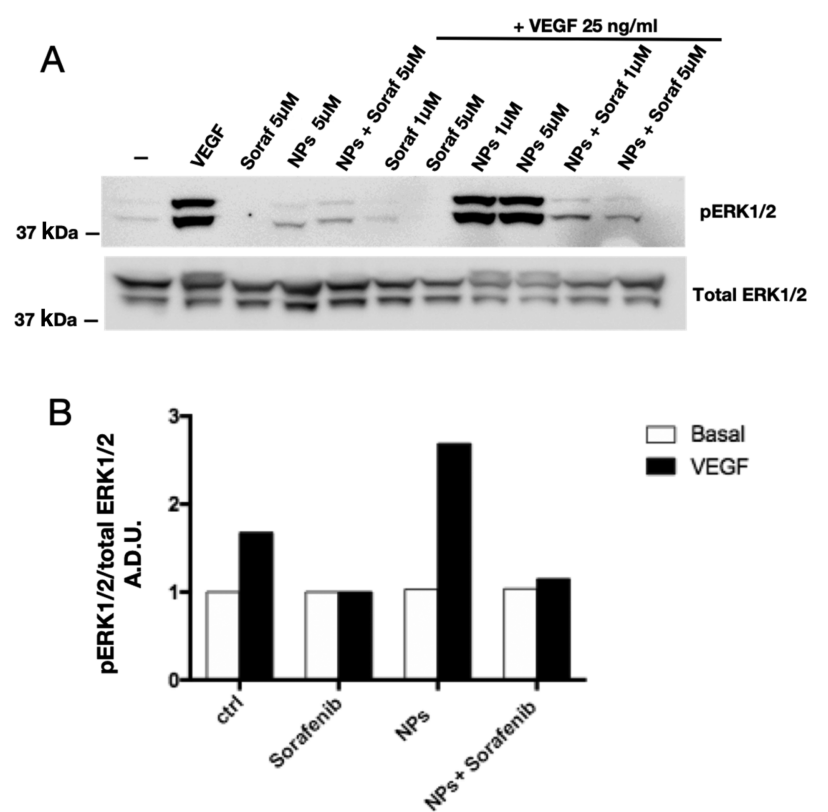

Figure 9. Effect of Sorafenib-loaded NPs on VEGF signaling in HUVEC. (A) Western blot analysis of ERK1/2 phosphorylation in HUVEC protein lysates. Cells were pretreated with Sorafenib (1 and $5 \mu \mathrm{M}$ ) and NPs and SRF-PluS NPs (diluted to obtain the similar final concentration of Sorafenib) for $6 \mathrm{~h}$, with or without VEGF (25 ng/ $\mathrm{mL}, 10 \mathrm{~min}$ ). (B) Ratio of arbitrary densitometric units (A.D.U.) of phospho-ERK1/2 to total ERK1/2.

that the drug, once released, completely prevents endothelial cell responses and postreceptor MAPK signaling ignited by VEGF, one of the major players of tumor angiogenesis. Our in vitro data document that this innovative preparation is safe, but only animal studies will definitely document both its safety and efficacy in models of pathology, parameters strongly necessary before any use in the clinic. The versatility of the synthesis of PluS NPs can also allow their doping with NIR emitting dyes, as cyanines, possibly yielding nanostructures able to combine optical imaging, photoacoustic imaging, drug delivery, and photothermal therapy, further increasing their theranostic potential.

\section{EXPERIMENTAL SECTION}

The UV-vis spectra were recorded with a PerkinElmer Lambda 45 spectrophotometer. The fluorescence spectra were recorded with a PerkinElmer LS55 spectrofluorimeter. All of the samples were placed in a quartz cuvette with $1 \mathrm{~cm}$ optical path length. Hydrodynamic diameters and size distributions of PluS NPs, Sorafenib-loaded PluS NPs (SRFPluS NPs), and SRF-F127 micelles were determined using dynamic light scattering (DLS). DLS measurements were carried out using a Malvern Zetasizer Nanoseries equipped with a $633 \mathrm{~nm}$ laser. All DLS measurements were performed in PBS ( $\mathrm{pH} 7.4)$ at $25^{\circ} \mathrm{C}$ and at a scattering angle of $173^{\circ}$. For each sample, mean hydrodynamic sizes and polydispersity index (PDI) were determined from three measurements of the autocorrelation function using cumulant analysis. Reported mean hydrodynamic diameters for each condition were determined by averaging values from triplicate measurements. Errors on mean hydrodynamic diameters were calculated from the standard deviation (SD) of values obtained from triplicate measurements.
Materials. Reagents and solvents were used as received without further purification: dimethyl sulfoxide (DMSO, 98\%, reagent grade, Sigma), methanol (98\%, reagent grade, Sigma), water (Milli-Q $18.2 \mu \mathrm{S}$ ), tetramethyl orthosilicate (TMOS, 98\%, Sigma), Sorafenib tosylate (SRF, 99\%, Santa Cruz Biotech), Pluronic F-127 [avg. molecular weight $(\mathrm{MW})=12$ $600 \mathrm{~g} / \mathrm{mol}$, Bioreagent, Sigma, F-127], 3-isocyanatopropyltrimethoxysilane (95\%, Gelest), poly(vinylpyrrolidone) K30 (avg. $\mathrm{MW}=40000 \mathrm{~g} / \mathrm{mol}$, Sigma, PVP), triethylamine (TEA, reagent grade, Sigma), Sephadex G-25 (Sigma), and phosphate-buffered saline (PBS, tablet, Sigma).

Rhodamine B Trimethoxysilane Derivative Synthesis. Rhodamine B piperazine derivative $\left(10 \mathrm{mg}, 1.9 \times 10^{-5} \mathrm{~mol}, 1\right.$ equiv $)^{41}$ was dissolved in a $1.5 \mathrm{~mL}$ plastic tube containing 0.5 $\mathrm{mL}$ of DMSO. Then, $3.3 \times 10^{-6} \mathrm{~L} 3$-isocyanatopropyltrimethoxysilane $\left(1.9 \times 10^{-5} \mathrm{~mol}, 1\right.$ equiv) and $6 \times 10^{-6} \mathrm{~L}$ TEA $\left(3.8 \times 10^{-5}\right.$ mol, 2 equiv) were added. The reaction mixture was stirred for $30 \mathrm{~min}$ and then used directly for the nanoparticle preparation without any further purification.

Synthesis of PluS NPs Loaded with Sorafenib (SRFPluS NPs). The synthesis of Sorafenib tosylate-loaded silica nanoparticles (SRF-PluS NPs) was carried out adapting previously reported strategies. ${ }^{42,43}$ The process started with the dissolution in a $20 \mathrm{~mL}$ scintillation vial containing $2 \mathrm{~mL}$ of methanol, $19 \mathrm{mg}$ of Sorafenib tosylate $\left(2.98 \times 10^{-5} \mathrm{~mol} \mathrm{SRF}-\right.$ NPs, $0 \mathrm{mg}$ for control NPs), and $200 \mathrm{mg}$ of Pluronic F-127. The organic solvent was removed under vacuum, and a welldried solid was recovered. This solid was dissolved in $3.2 \mathrm{~mL}$ of PBS ( $\mathrm{pH}$ 7.4). After the complete dissolution of the surfactant and of the drug, $50 \mu \mathrm{L}$ of a $38 \mathrm{mM}$ DMSO solution of rhodamine $\mathrm{B}$ trimethoxysilane derivative was added [RB$\left.\mathrm{Si}(\mathrm{OMe})_{3}\right]$. Then, $250 \mu \mathrm{L}$ of TMOS $\left(1.68 \times 10^{-3} \mathrm{~mol}\right)$ was added and the reaction mixture was stirred at room temperature for $45 \mathrm{~min}$. A volume of $0.5 \mathrm{~mL}$ of NPs suspension was purified by size exclusion chromatography (SEC) using Sephadex G-25 (8 mL), obtaining a final volume of $1.5 \mathrm{~mL}$.

NPs Freeze-Drying. To $1.5 \mathrm{~mL}$ of the purified nanoparticle solution, $100 \mu \mathrm{L}$ of PVP K30 (10 wt \%) was added as a stabilizing agent for the freeze-drying step. The solution, then, was immediately frozen with liquid nitrogen and then freezedried for $24 \mathrm{~h}$ at $-50{ }^{\circ} \mathrm{C}$ under vacuum (about $10^{-3} \mathrm{~atm}$ ) using a $\mathrm{LABCO}$ freeze dryer. At the end of the dehydration procedure, a fine pink powder was recovered and stored at about $-18{ }^{\circ} \mathrm{C}$. The nanoparticles resuspended even after a period of 6 months after the freeze-drying maintained their colloidal and drug release properties. The amount of SRF contained in $1 \mathrm{mg}$ of lyophilized solid corresponds to $25 \mu \mathrm{g}$ (drug loading has been observed to be constant up to 6 months after the preparation). The final averaged dye and drug doping degree consist of six rhodamine B and 45 SRF molecules per nanoparticle, respectively.

Estimation of the Nanoparticle Dye/Drug content. The average number of dyes per particle was computed as previously reported, ${ }^{30,42}$ considering the molar extinction coefficient of the nanoparticles at $\lambda=565 \mathrm{~nm}$ (rhodamine $\mathrm{B}$ signal) before and after the lyophilization and dividing this value by the molar extinction coefficient of rhodamine $\mathrm{B}$ $\left(\varepsilon_{\mathrm{EtOH}, 565 \mathrm{~nm}}=1 \times 10^{5} \mathrm{M}^{-1} \mathrm{~cm}^{-1}\right)$. The concentration of NPs obtained with this synthetic procedure has been evaluated with different experimental techniques as previously reported. ${ }^{42}$ The signal of the rhodamine $\mathrm{B}$ in the nanoparticle dispersion was used as an internal reference to compute the average number 
of Sorafenib molecules physically entrapped inside the NP, considering the molar extinction coefficient of the drug in methanol $\left(\varepsilon_{\mathrm{MeOH}, 267 \mathrm{~nm}}=3.9 \times 10^{4} \mathrm{M}^{-1} \mathrm{~cm}^{-1}\right)$.

Drug Release Kinetics. UV-vis analysis was used to evaluate the release of Sorafenib from the nanoparticles. The UV-vis spectrum of the drug shows a narrow band centered at $265 \mathrm{~nm}$ only when perfectly solubilized (as in the case of organic solvent solutions, see Figures S5 and S6, SI). When SRF is dispersed in water (even at $1 \times 10^{-6} \mathrm{M}$ ), the $265 \mathrm{~nm}$ absorption band becomes less intense with respect to that shown in an organic solvent. This spectral behavior can be due to the aggregation of Sorafenib in aqueous solutions since this drug is insoluble in water (Figure S5). Experimentally, the kinetics were recorded with a Varian Cary 50 Bio spectrophotometer. A small amount of lyophilized SRF-PluS NPs or of SRF and F-127 (SRF-F127) was dispersed to the same final concentration of Sorafenib $\left(1.1 \times 10^{-5} \mathrm{M}\right)$ and of the carrier $\left(2.5 \times 10^{-7} \mathrm{M}\right)$ with PBS $(\mathrm{pH} 7.4)$ in a $1 \mathrm{~cm}$ optical path quartz cuvette. The carrier was the NP or a comparable amount of Pluronic F-127 surfactant. During the acquisition of the kinetic experiments, the solution was stirred at $1000 \mathrm{rpm}$ at $37{ }^{\circ} \mathrm{C}$. A UV-vis absorption spectrum each for $10 \mathrm{~min}$ for SRF-F127 and $45 \mathrm{~min}$ for SRF-NPs was recorded in the wavelength range $240-750 \mathrm{~nm}$ (scan speed $480 \mathrm{~nm} / \mathrm{min}$ ).

Cell Cultures. Human umbilical vein endothelial cells (HUVECs) were purchased from Promocell (Heidelberg, Germany) and were grown in endothelial growth medium-2, containing VEGF, $\mathrm{R}^{3}$-IGF-1, hEGF, hFGF, hydrocortisone, ascorbic acid, heparin, and GA-1000 (Lonza, Basel, Switzerland); $10 \%$ fetal bovine serum (FBS) (Hyclone, EuroClone, Milan, Italy); and $2 \mathrm{mM}$ glutamine, 100 units/mL penicillin, and $0.1 \mathrm{mg} / \mathrm{mL}$ streptomycin (Merck KGaA, Darmstadt, Germany). Cells were cultured at $37{ }^{\circ} \mathrm{C}$ in $5 \% \mathrm{CO}_{2}$ and used until passage 10 .

We have tried to characterize the nanoparticle hydrodynamic size in serum, but, in this medium, the scattering of proteins is so strong that it hides the nanoparticle scattering signal. At the same time, the presence of large concentrations of proteins and of their aggregates makes the DLS technique unsuitable to perform a reliable characterization since the level of polydispersity of the suspension is too high. However, in previous works, ${ }^{32,33}$ we investigated the colloidal behavior of Pluronic silica nanoparticles in PBS in the presence of increasing concentration of bovine serum albumin (BSA) (up to $6 \mathrm{mg} / \mathrm{mL}$ ). In these conditions, we observed that the Pluronic silica nanoparticles maintain their colloidal stability and monodispersity, experimenting a 2-4-fold increase in their hydrodynamic diameter (BSA concentration range $0-5.6 \% \mathrm{w} /$ $\mathrm{v})$, depending on the surface architecture of the Pluronic silica nanoparticle.

Experimental Design. The experiments were designed to evaluate the effect of Sorafenib-loaded PluS NPs having Sorafenib concentrations at 1 and $5 \mu \mathrm{M}$. The experimental controls were represented by no treatment (control condition, Ctrl), NPs alone (at the same concentration as the ones loaded with the drug, NPs), and free drug (Sorafenib, 1 or $5 \mu \mathrm{M}$ ). Two types of NP preparations were used: the first one freshly prepared before cell treatment and the second one with lyophilized NPs, reconstituted in medium before cell challenging.

Immunofluorescence Analysis. The cytoskeletal and intermediate filament proteins $\beta$-actin and vimentin were monitored by fluorescence analysis. A total of $5 \times 10^{4}$
HUVECs were seeded on $1 \mathrm{~cm}$ circular glass coverslips placed into 24-well Multiwell plates and then exposed to NPs (at the concentration corresponding to $5 \mu \mathrm{M}$ Sorafenib) in medium containing 1\% FBS. After $24 \mathrm{~h}$ incubation, cells were fixed in acetone for $5 \mathrm{~min}$. After blocking of unspecific bindings with $3 \%$ bovine serum albumin (BSA), cells were incubated overnight at $4^{\circ} \mathrm{C}$ with the primary antibodies [anti $\beta$-actin (1:70, Sigma) and anti-vimentin (1:50, Cell Signaling, Milan, Italy)] in $0.5 \%$ BSA in PBS. Samples were then incubated with a secondary antibody tetramethylrhodamine conjugated (Sigma) in PBS with $0.5 \%$ BSA for 90 min. Coverslips were mounted in fluoromount (Sigma-Aldrich), and pictures of stained cells were taken by fluorescence microscopy at $63 x$ magnification. ${ }^{44}$

Cell Number and Morphology. Cells were seeded at a density of $2.5 \times 10^{4}$ on immunofluorescence coverslips located in 24-well Multiwell plates. After $24 \mathrm{~h}$, NPs with and without Sorafenib ( 1 and $5 \mu \mathrm{M}$ ) were added to culture medium containing 1 or $5 \%$ serum. Where indicated, cells were stimulated with VEGF $(25 \mathrm{ng} / \mathrm{mL})$ in medium with $0.1 \%$ FBS. After 24, 48, or $72 \mathrm{~h}$ of incubation, cells were fixed with $100 \%$ methanol and stained with hematoxylin and eosin to monitor the cell morphology and number. Living and attached cells were counted randomly in 10 fields/sample at $20 \times$ original magnification as previously reported. ${ }^{45}$

Scratch Assay. HUVECs $\left(1 \times 10^{5}\right.$ cells $)$ were seeded on the bottom of 12-well Multiwell plates precoated with gelatin. Once HUVEC reached the confluence, cells were scratched using a sterile $100-1000 \mu \mathrm{L}$ micropipette tip to create a wound $\pm 500 \mu \mathrm{m}$ across the monolayer. Each well was washed with PBS to remove detached cells. Cells were exposed to NPs with and without Sorafenib $(5 \mu \mathrm{M})$ in medium with 1 and $5 \%$ FBS. Where indicated, cells were stimulated with VEGF (25 $\mathrm{ng} / \mathrm{mL}$ ) in medium with $0.1 \%$ FBS after a $6 \mathrm{~h}$ preincubation with NPs with and without Sorafenib $(5 \mu \mathrm{M})$. The antimitotic ARA-C $(2.5 \mu \mathrm{g} / \mathrm{mL})$ was added in all of the transwells to evaluate only migration. Images of the wound in each well were acquired from 0 to $18 \mathrm{~h}$ using a phase contrast microscope (Nikon Eclipse TE 300, Nikon, Tokyo, Japan) at $20 \times$ magnification. The rate of migration was measured by quantifying the uncovered area of the wound that HUVEC covered starting from the edge of the scratch. Results are expressed as the percentage of the wound area. ${ }^{45}$

Tube Formation Assay. Endothelial cells were plated on solidified Matrigel $\left(1.5 \times 10^{5}\right.$ cells in a 12 -well Multiwell plate). After $18 \mathrm{~h}$ of incubation with NPs with and without Sorafenib $(5 \mu \mathrm{M})$ in medium with $5 \%$ FBS, endothelial cells were photographed and network formation on Matrigel was measured by means of the number of circles (Nikon Eclipse E400 and Nikon DS-5MC camera). ${ }^{45}$

Western Blotting for ERK1/2 Phosphorylation. Phosphorylation of ERK1/2 was evaluated by western blotting as previously described. ${ }^{4,45}$ Sparse and serum-starved HUVECs seeded in $60 \mathrm{~mm}$ Petri dishes were pretreated for 1 or $6 \mathrm{~h}$ with NPs with or without Sorafenib (1 and $5 \mu \mathrm{M})$ before the stimulation with $25 \mathrm{ng} / \mathrm{mL}$ VEGF for $10 \mathrm{~min}$. Cytosolic fractions were obtained by the use of lysis buffer with the following composition: $50 \mathrm{mM}$ Tris- $\mathrm{HCl}, 1 \%$ Triton-X, $1 \mathrm{mM}$ $\mathrm{Na}_{3} \mathrm{VO}_{4}, 1 \mathrm{mM}$ ethylene glycol-bis $(\beta$-aminoethyl ether)$N, N, N^{\prime}, N^{\prime}$-tetraacetic acid, $0.2 \mathrm{mM}$ phenylmethanesulfonyl fluoride, $25 \mu \mathrm{g} / \mathrm{mL}$ leupeptin, $10 \mu \mathrm{g} / \mathrm{mL}$ aprotinin, $10 \mathrm{mM}$ $\mathrm{NaF}$, and $150 \mathrm{mM} \mathrm{NaCl}$. After centrifugation at $16000 \mathrm{~g}$ for $20 \mathrm{~min}$ at $4{ }^{\circ} \mathrm{C}$, the supernatant was collected and proteins 
were assayed. Electrophoresis was carried out in 4-12\% BisTris gels (Life Technologies, Carlsbad, CA). Proteins were then blotted onto activated nitrocellulose membranes, incubated overnight with the antibody anti phospho-ERK1/2 (Cell Signalling Technology, Euroclone, Pero, Milan, Italy) diluted 1:1000 in PBS containing 1\% dried milk and $0.05 \%$ Tween 20, and then detected by an enhanced chemiluminescence system (GE Healthcare, Milan, Italy). Results were normalized to those obtained using an antibody against total ERK1/2 (Cell Signalling Technology, Euroclone, Pero, Milan, Italy).

Immunoblots were analyzed by densitometry using NIH Image J $1.48 \mathrm{v}$ software, and the results, expressed as arbitrary density units (A.D.U.) $\pm \mathrm{SD}$, were normalized to $\beta$-actin.

Data Analysis and Statistical Procedures. Results are either representative or the average of at least three independent experiments done in triplicate. Statistical analysis was performed using analysis of the variance test followed by the Bonferroni test and the Student $t$ test (when appropriate), using GraphPad Prism version 4.00 for Windows (GraphPad Software, La Jolla, CA). $p<0.05$ was considered statistically significant.

\section{ASSOCIATED CONTENT}

\section{S Supporting Information}

The Supporting Information is available free of charge on the ACS Publications website at DOI: 10.1021/acsomega.9b01699.

Photophysical characterization of Sorafenib; morphological and photophysical characterization of the PluS NPs; and cytotoxicity evaluation of PVP on HUVEC cells (PDF)

\section{AUTHOR INFORMATION}

\section{Corresponding Authors}

*E-mail: luca.prodi@unibo.it (L.P.).

*E-mail: lucia.morbidelli@unisi.it (L.M.).

ORCID $\odot$

Damiano Genovese: 0000-0002-4389-7247

Luca Prodi: 0000-0002-1630-8291

\section{Author Contributions}

The manuscript was written through contributions of all authors. All authors have given approval to the final version of the manuscript.

\section{Funding}

This research was funded by the Italian Ministry of Education, University and Research (MIUR) PRIN project no. 2015Y3C5KP to L.M. and PRIN project no. 2017EKCS35 to L.P.

\section{Notes}

The authors declare no competing financial interest.

\section{ACKNOWLEDGMENTS}

Technical assistance by Federica Finetti, Lorenzo Bazzani, and Inesa Hyseni is gratefully acknowledged.

\section{ABBREVIATIONS}

AEs, adverse events; BSA, bovine serum albumin; DLS, dynamic light scattering; EPR, enhanced permeability and retention; FBS, fetal bovine serum; HUVEC, human umbilical vein endothelial cell; NPs, nanoparticles; SRF-PluS NPs,
Sorafenib-loaded PluS NPs; TEOS, tetraethylorthosilicate; TMOS, tetramethoxyorthosilicate; TKIs, tyrosine kinase inhibitors; VEGF, vascular endothelial growth factor; VEGFR, vascular endothelial growth factor receptor

\section{REFERENCES}

(1) Escudier, B.; Worden, F.; Kudo, M. Sorafenib: key lessons from over 10 years of experience. Expert Rev. Anticancer Ther. 2019, 177189.

(2) Cook, K. M.; Figg, W. D. Angiogenesis inhibitors: current strategies and future prospects. CA-Cancer J. Clin. 2010, 60, 222243.

(3) Weis, S. M.; Cheresh, D. A. Tumor angiogenesis: molecular pathways and therapeutic targets. Nat. Med. 2011, 17, 1359-1370.

(4) Zhong, H.; Bowen, J. P. Recent advances in small molecule inhibitors of VEGFR and EGFR signaling pathways. Curr. Top. Med. Chem. 2011, 11, 1571-1590.

(5) Wild, A. T.; Gandhi, N.; Chettiar, S. T.; Aziz, K.; Gajula, R. P.; Williams, R. D.; Kumar, R.; Taparra, K.; Zeng, J.; Cades, J. A.; Velarde, E.; Menon, S.; Geschwind, J. F.; Cosgrove, D.; Pawlik, T. M.; Maitra, A.; Wong, J.; Hales, R. K.; Torbenson, M. S.; Herman, J. M.; Tran, P. T. Concurrent versus sequential sorafenib therapy in combination with radiation for hepatocellular carcinoma. PLoS One 2013, 8, No. e65726.

(6) Xie, B.; Wang, D. H.; Spechler, S. J. Sorafenib for treatment of hepatocellular carcinoma: a systematic review. Dig. Dis. Sci. 2012, 57, $1122-1129$.

(7) Escudier, B.; Eisen, T.; Stadler, W. M.; Szczylik, C.; Oudard, S.; Staehler, M.; Negrier, S.; Chevreau, C.; Desai, A. A.; Rolland, F.; Demkow, T.; Hutson, T. E.; Gore, M.; Anderson, S.; Hofilena, G.; Shan, M.; Pena, C.; Lathia, C.; Bukowski, R. M. Sorafenib for treatment of renal cell carcinoma: Final efficacy and safety results of the phase III treatment approaches in renal cancer global evaluation trial. J. Clin. Oncol. 2009, 27, 3312-3318.

(8) Flaherty, K. T.; Lee, S. J.; Zhao, F.; Schuchter, L. M.; Flaherty, L.; Kefford, R.; Atkins, M. B.; Leming, P.; Kirkwood, J. M. Phase III trial of carboplatin and paclitaxel with or without sorafenib in metastatic melanoma. J. Clin. Oncol. 2013, 31, 373-379.

(9) Gupta-Abramson, V.; Troxel, A. B.; Nellore, A.; Puttaswamy, K.; Redlinger, M.; Ransone, K.; Mandel, S. J.; Flaherty, K. T.; Loevner, L. A.; O'Dwyer, P. J.; Brose, M. S. Phase II trial of sorafenib in advanced thyroid cancer. J. Clin. Oncol. 2008, 26, 4714-4719.

(10) Maki, R. G.; D’Adamo, D. R.; Keohan, M. L.; Saulle, M.; Schuetze, S. M.; Undevia, S. D.; Livingston, M. B.; Cooney, M. M.; Hensley, M. L.; Mita, M. M.; Takimoto, C. H.; Kraft, A. S.; Elias, A. D.; Brockstein, B.; Blachere, N. E.; Edgar, M. A.; Schwartz, L. H.; Qin, L. X.; Antonescu, C. R.; Schwartz, G. K. Phase II study of sorafenib in patients with metastatic or recurrent sarcomas. J. Clin. Oncol. 2009, 27, 3133-3140.

(11) Printz, C. Clinical trials of note. Sorafenib as adjuvant treatment in the prevention of disease recurrence in patients with hepatocellular carcinoma (HCC) (STORM). Cancer 2009, 115, 4646.

(12) Ravandi, F.; Alattar, M. L.; Grunwald, M. R.; Rudek, M. A.; Rajkhowa, T.; Richie, M. A.; Pierce, S.; Daver, N.; Garcia-Manero, G.; Faderl, S.; Nazha, A.; Konopleva, M.; Borthakur, G.; Burger, J.; Kadia, T.; Dellasala, S.; Andreeff, M.; Cortes, J.; Kantarjian, H.; Levis, M. Phase 2 study of azacytidine plus sorafenib in patients with acute myeloid leukemia and FLT-3 internal tandem duplication mutation. Blood 2013, 121, 4655-4662.

(13) Funakoshi, T.; Latif, A.; Galsky, M. D. Risk of hypertension in cancer patients treated with sorafenib: an updated systematic review and meta-analysis. J. Hum. Hypertens. 2013, 27, 601-611.

(14) Choueiri, T. K.; Schutz, F. A.; Je, Y.; Rosenberg, J. E.; Bellmunt, J. Risk of arterial thromboembolic events with sunitinib and sorafenib: a systematic review and meta-analysis of clinical trials. J. Clin. Oncol. 2010, 28, 2280-2285.

(15) Qi, W. X.; Min, D. L.; Shen, Z.; Sun, Y. J.; Lin, F.; Tang, L. N.; He, A. N.; Yao, Y. Risk of venous thromboembolic events associated 
with VEGFR-TKIs: a systematic review and meta-analysis. Int. J. Cancer 2013, 132, 2967-2974.

(16) Sivendran, S.; Liu, Z.; Portas, L. J., Jr.; Yu, M.; Hahn, N.; Sonpavde, G.; Oh, W. K.; Galsky, M. D. Treatment-related mortality with vascular endothelial growth factor receptor tyrosine kinase inhibitor therapy in patients with advanced solid tumors: a metaanalysis. Cancer Treat. Rev. 2012, 38, 919-925.

(17) Je, Y.; Schutz, F. A.; Choueiri, T. K. Risk of bleeding with vascular endothelial growth factor receptor tyrosine-kinase inhibitors sunitinib and sorafenib: a systematic review and meta-analysis of clinical trials. Lancet Oncol. 2009, 10, 967-974.

(18) Pouessel, D.; Culine, S. High frequency of intracerebral hemorrhage in metastatic renal carcinoma patients with brain metastases treated with tyrosine kinase inhibitors targeting the vascular endothelial growth factor receptor. Eur. Urol. 2008, 53, 376-381.

(19) Pelaz, B.; Alexiou, C.; Alvarez-Puebla, R. A.; Alves, F.; Andrews, A. M.; Ashraf, S.; Balogh, L. P.; Ballerini, L.; Bestetti, A.; Brendel, C.; Bosi, S.; Carril, M.; Chan, W. C. W.; Chen, C.; Chen, X.; Chen, X.; Cheng, Z.; Cui, D.; Du, J.; Dullin, C.; Escudero, A.; Feliu, N.; Gao, M.; George, M.; Gogotsi, Y.; Grünweller, A.; Gu, Z.; Halas, N. J.; Hampp, N.; Hartmann, R. K.; Hersam, M. C.; Hunziker, P.; Jian, J.; Jiang, X.; Jungebluth, P.; Kadhiresan, P.; Kataoka, K.; Khademhosseini, A.; Kopeček, J.; Kotov, N. A.; Krug, H. F.; Lee, D. S.; Lehr, C.-M.; Leong, K. W.; Liang, X.-J.; Ling Lim, M.; LizMarzán, L. M.; Ma, X.; Macchiarini, P.; Meng, H.; Möhwald, H.; Mulvaney, P.; Nel, A. E.; Nie, S.; Nordlander, P.; Okano, T.; Oliveira, J.; Park, T. H.; Penner, R. M.; Prato, M.; Puntes, V.; Rotello, V. M.; Samarakoon, A.; Schaak, R. E.; Shen, Y.; Sjöqvist, S.; Skirtach, A. G.; Soliman, M. G.; Stevens, M. M.; Sung, H.-W.; Tang, B. Z.; Tietze, R.; Udugama, B. N.; VanEpps, J. S.; Weil, T.; Weiss, P. S.; Willner, I.; Wu, Y.; Yang, L.; Yue, Z.; Zhang, Q.; Zhang, Q.; Zhang, X.-E.; Zhao, Y.; Zhou, X.; Parak, W. J. Diverse Applications of Nanomedicine. ACS Nano 2017, 11, 2313-2381.

(20) Baetke, S. C.; Lammers, T.; Kiessling, F. Applications of nanoparticles for diagnosis and therapy of cancer. Br. J. Radiol. 2015, 88, No. 20150207.

(21) Greish, K. Enhanced permeability and retention (EPR) effect for anticancer nanomedicine drug targeting. Methods Mol. Biol. 2010, $624,25-37$.

(22) Nakamura, Y.; Mochida, A.; Choyke, P. L.; Kobayashi, H. Nanodrug Delivery: Is the Enhanced Permeability and Retention Effect Sufficient for Curing Cancer? Bioconjugate Chem. 2016, 27, 2225-2238.

(23) Ma, M.; Chen, H.; Chen, Y.; Zhang, K.; Wang, X.; Cui, X.; Shi, J. Hyaluronic acid-conjugated mesoporous silica nanoparticles: excellent colloidal dispersity in physiological fluids and targeting efficacy. J. Mater. Chem. 2012, 22, 5615-5621.

(24) Montalti, M.; Prodi, L.; Rampazzo, E.; Zaccheroni, N. Dyedoped silica nanoparticles as luminescent organized systems for nanomedicine. Chem. Soc. Rev. 2014, 43, 4243-4268.

(25) Rampazzo, E.; Genovese, D.; Palomba, F.; Prodi, L.; Zaccheroni, N. NIR-fluorescent dye doped silica nanoparticles for in vivo imaging, sensing and theranostic. Methods Appl. Fluoresc. 2018, 6, No. 022002.

(26) Bradbury, M. S.; Phillips, E.; Montero, P. H.; Cheal, S. M.; Stambuk, H.; Durack, J. C.; Sofocleous, C. T.; Meester, R. J.; Wiesner, U.; Patel, S. Clinically-translated silica nanoparticles as dual-modality cancer-targeted probes for image-guided surgery and interventions. Integr. Biol. 2013, 5, 74-86.

(27) Maggini, L.; Travaglini, L.; Cabrera, I.; Castro-Hartmann, P.; De Cola, L. Biodegradable Peptide-Silica Nanodonuts. Chem. - Eur. J. 2016, 22, 3697-3703.

(28) Maggini, L.; Cabrera, I.; Ruiz-Carretero, A.; Prasetyanto, E. A.; Robinet, E.; De Cola, L. Breakable mesoporous silica nanoparticles for targeted drug delivery. Nanoscale 2016, 8, 7240-7247.

(29) Genovese, D.; Bonacchi, S.; Juris, R.; Montalti, M.; Prodi, L.; Rampazzo, E.; Zaccheroni, N. Prevention of Self-Quenching in
Fluorescent Silica Nanoparticles by Efficient Energy Transfer. Angew. Chem., Int. Ed. 2013, 52, 5965-5968.

(30) Rampazzo, E.; Bonacchi, S.; Genovese, D.; Juris, R.; Montalti, M.; Paterlini, V.; Zaccheroni, N.; Dumas-Verdes, C.; Clavier, G.; Méallet-Renault, R.; Prodi, L. Pluronic-Silica (PluS) Nanoparticles Doped with Multiple Dyes Featuring Complete Energy Transfer. J. Phys. Chem. C 2014, 118, 9261-9267.

(31) Rampazzo, E.; Voltan, R.; Petrizza, L.; Zaccheroni, N.; Prodi, L.; Casciano, F.; Zauli, G.; Secchiero, P. Proper design of silica nanoparticles combines high brightness, lack of cytotoxicity and efficient cell endocytosis. Nanoscale 2013, 5, 7897-7905.

(32) Rampazzo, E.; Boschi, F.; Bonacchi, S.; Juris, R.; Montalti, M.; Zaccheroni, N.; Prodi, L.; Calderan, L.; Rossi, B.; Becchi, S.; Sbarbati, A. Multicolor core/shell silica nanoparticles for in vivo and ex vivo imaging. Nanoscale 2012, 4, 824-830.

(33) Helle, M.; Rampazzo, E.; Monchanin, M.; Marchal, F.; Guillemin, F.; Bonacchi, S.; Salis, F.; Prodi, L.; Bezdetnaya, L. Surface Chemistry Architecture of Silica Nanoparticles Determine the Efficiency of in Vivo Fluorescence Lymph Node Mapping. ACS Nano 2013, 7, 8645-8657.

(34) Biffi, S.; Petrizza, L.; Garrovo, C.; Rampazzo, E.; Andolfi, L.; Giustetto, P.; Nikolov, I.; Kurdi, G.; Danailov, M. B.; Zauli, G.; Secchiero, P.; Prodi, L. Multimodal near-infrared-emitting PluS Silica nanoparticles with fluorescent, photoacoustic, and photothermal capabilities. Int. J. Nanomed. 2016, 11, 4865-4874.

(35) Rampazzo, E.; Bonacchi, S.; Juris, R.; Genovese, D.; Prodi, L.; Zaccheroni, N.; Montalti, M. Dual-Mode, Anisotropy-Encoded, Ratiometric Fluorescent Nanosensors: Towards Multiplexed Detection. Chem. - Eur. J. 2018, 24, 16743-16746.

(36) Valenti, G.; Rampazzo, E.; Bonacchi, S.; Petrizza, L.; Marcaccio, M.; Montalti, M.; Prodi, L.; Paolucci, F. Variable Doping Induces Mechanism Swapping in Electrogenerated Chemiluminescence of $\mathrm{Ru}(\text { bpy })_{3}{ }^{2+}$ Core-Shell Silica Nanoparticles. J. Am. Chem. Soc. 2016, $138,15935-15942$.

(37) Abdelwahed, W.; Degobert, G.; Stainmesse, S.; Fessi, H. Freeze-drying of nanoparticles: formulation, process and storage considerations. Adv. Drug Delivery Rev. 2006, 58, 1688-1713.

(38) Picco, A. S.; Ferreira, L. F.; Liberato, M. S.; Mondo, G. B.; Cardoso, M. B. Freeze-drying of silica nanoparticles: redispersibility toward nanomedicine applications. Nanomedicine 2018, 13, 179-190.

(39) Moore, C. J.; Montón, H.; O’Kennedy, R.; Williams, D. E.; Nogués, C.; Crean, C.; Gubala, V. Controlling colloidal stability of silica nanoparticles during bioconjugation reactions with proteins and improving their longer-term stability, handling and storage. J. Mater. Chem. B 2015, 3, 2043-2055.

(40) Mody, K. T.; Mahony, D.; Cavallaro, A. S.; Stahr, F.; Qiao, S. Z.; Mahony, T. J.; Mitter, N. Freeze-drying of ovalbumin loaded mesoporous silica nanoparticle vaccine formulation increases antigen stability under ambient conditions. Int. J. Pharm. 2014, 465, 325-332.

(41) Nguyen, T.; Francis, M. B. Practical Synthetic Route to Functionalized Rhodamine Dyes. Org. Lett. 2003, 5, 3245-3248.

(42) Rampazzo, E.; Bonacchi, S.; Juris, R.; Montalti, M.; Genovese, D.; Zaccheroni, N.; Prodi, L.; Rambaldi, D. C.; Zattoni, A.; Reschiglian, P. Energy Transfer from Silica Core-Surfactant Shell Nanoparticles to Hosted Molecular Fluorophores. J. Phys. Chem. B 2010, 114, 14605-14613.

(43) Valenti, G.; Rampazzo, E.; Bonacchi, S.; Khajvand, T.; Juris, R.; Montalti, M.; Marcaccio, M.; Paolucci, F.; Prodi, L. A versatile strategy for tuning the color of electrochemiluminescence using silica nanoparticles. Chem. Commun. 2012, 48, 4187-4189.

(44) Finetti, F.; Terzuoli, E.; Donnini, S.; Uva, M.; Ziche, M.; Morbidelli, L. Monitoring Endothelial and Tissue Responses to Cobalt Ferrite Nanoparticles and Hybrid Hydrogels. PLoS One 2016, 11, No. e0168727.

(45) Terzuoli, E.; Monti, M.; Vellecco, V.; Bucci, M.; Cirino, G.; Ziche, M.; Morbidelli, L. Characterization of zofenoprilat as an inducer of functional angiogenesis through increased $\mathrm{H} 2 \mathrm{~S}$ availability. Br. J. Pharmacol. 2015, 172, 2961-2973. 
(46) Arca, M.; Caltagirone, C.; De Filippo, G.; Formica, M.; Fusi, V.; Giorgi, L.; Lippolis, V.; Prodi, L.; Rampazzo, E.; Scorciapino, M. A.; Sgarzi, M.; Zaccheroni, N. A fluorescent ratiometric nanosized system for the determination of $\mathrm{Pd}(\mathrm{II})$ in water. Chem. Commun. 2014, 50, 15259-15262.

(47) Bazzicalupi, C.; Caltagirone, C.; Cao, Z.; Chen, Q.; Di Natale, C.; Garau, A.; Lippolis, V.; Lvova, L.; Liu, H.; Lundstrom, I.; Mostallino, M. C.; Nieddu, M.; Paolesse, R.; Prodi, L.; Sgarzi, M.; Zaccheroni, N. Multimodal use of new coumarin-based fluorescent chemosensors: towards highly selective optical sensors for $\mathrm{Hg}(2+)$ probing. Chem. - Eur. J. 2013, 19, 14639-14653.

(48) Palomba, F.; Genovese, D.; Petrizza, L.; Rampazzo, E.; Zaccheroni, N.; Prodi, L. Mapping heterogeneous polarity in multicompartment nanoparticles. Sci. Rep. 2018, 8, No. 17095.

(49) Genovese, D.; Montalti, M.; Prodi, L.; Rampazzo, E.; Zaccheroni, N.; Tosic, O.; Altenhöner, K.; May, F.; Mattay, J. Reversible photoswitching of dye-doped core-shell nanoparticles. Chem. Commun. 2011, 47, 10975-10977. 